\title{
Optimizing transport in a homogeneous network
}

\author{
Marc Durand* \\ DEAS, Harvard University, 29 Oxford Street, Cambridge, Massachusetts 02138, USA \\ Denis Weaire \\ Department of Physics, Trinity College Dublin, Dublin 2, Ireland \\ (Received 12 April 2004; published 28 October 2004)
}

\begin{abstract}
Many situations in physics, biology, and engineering consist of the transport of some physical quantity through a network of narrow channels. The ability of a network to transport such a quantity in every direction can be described by the average conductivity associated with it. When the flow through each channel is conserved and derives from a potential function, we show that there exists an upper bound of the average conductivity and explicitly give the expression for this upper bound as a function of the channel permeability and channel length distributions. Moreover, we express the necessary and sufficient conditions on the network structure to maximize the average conductivity. These conditions are found to be independent of the connectivity of the vertices.
\end{abstract}

DOI: 10.1103/PhysRevE.70.046125

\section{INTRODUCTION}

Examples of transport phenomena through a network of channels abound in nature and engineering: blood flow through the microcirculatory system, water transport through the venation of a leaf, water and electricity supply in a city, heat conduction through an open cell material, etc. Therefore, the search for a network structure optimizing the transport processes may be useful for a better understanding of the structure of natural networks and for the conception of optimized materials. Different functions can be optimized, such as dissipated power, volume, or surface area of the channels. Different constraints can be imposed: topology, flow rate, volume, etc. Various models have been proposed to understand the structure of natural networks [1,2], mostly based on the assumption of local optimization. The idea is to move the position of a given junction, with the other junctions and the topology fixed, in order to optimize one of the above functions. In a previous work, we considered the global optimization of the whole network structure for its electrical property [3]. We showed the existence of an upper bound for the average electrical conductivity of a network made of uniform wires, for a given amount of material per unit volume, and proved two necessary and sufficient conditions on the structure of the network for the upper bound to be reached. We then used these results to derive the expression of electrical conductivity of dry foams. In the present paper, we generalize the idea of an optimal structure for transport processes to networks made of non-uniform pipes and to any flow process, and derive three more general necessary and sufficient conditions for maximizing the transport properties. Surprisingly, we find that these conditions do not depend on the connectivity of the junctions.

Let us first denote each pipe by a pair of indices $(i, j)$ corresponding to the labels of its two ends. We consider pipes for which the aspect ratio is very large, so we can

*Electronic address: mdurand@deas.harvard.edu
PACS number(s): 02.50. - r, 89.75.Hc, 89.75.Fb

univocally define for each pipe $(i, j)$ a length $l_{i j}$, a local cross-sectional area $s_{i j}(l)$, and a local permeability $k_{i j}(l)$ $\left[k_{i j}(l)\right.$ is function of $s_{i j}(l)$ and both can vary with the curvilinear coordinate $l$ along the channel]. Let us assume that the flow through each channel is directly related to the gradient of a potential function $V$, so the flow vector $\mathbf{I}$, the permeability, and $V$ are related by

$$
\mathbf{I}=-k_{i j}(l) \boldsymbol{\nabla} V \text {. }
$$

Moreover, we suppose that the flow is in a steady state (so $V$ satisfies Laplace equation $\nabla^{2} V=0$ ) and is conserved through each pipe and each junction. We then define the dissipated power associated with the flow in the network as

$$
P=-\sum_{(i, j)} \int_{0}^{l_{i j}} \mathbf{I} \cdot \boldsymbol{\nabla} V d l .
$$

Using the assumption of steady-state flow, we can rewrite the dissipated power as

$$
P=\sum_{(i, j)} u_{i j} i_{i j}
$$

where $u_{i j}$ and $i_{i j}$ are, respectively, the potential difference and the flow rate through the channel $(i, j)$. Since the flow is conserved through each pipe and each junction, the total dissipated power in the network is equal to the product $U \times I$, where $U$ is the potential difference between the inlet (source) and the outlet (sink) of the network and $I$ is the crossing flow rate, as for the dissipated power of an electrical network. Pursuing the analogy, it follows that the actual distribution of flow rates for a given total flow rate is the one which minimizes the dissipated power, and subsequently the monotonicity law of Rayleigh is valid [3-5] : If any of the resistances of a circuit are increased, the effective resistance between any two points can only increase. If they are decreased, the effective resistance can only decrease. Although the monotonicity law cannot predict the sign of the effective resistance variation when some resistances are increased and some others are decreased (the variation obviously depends on the structure of the network), it will be helpful to determine the optimal geometry of a network for its transport properties. 


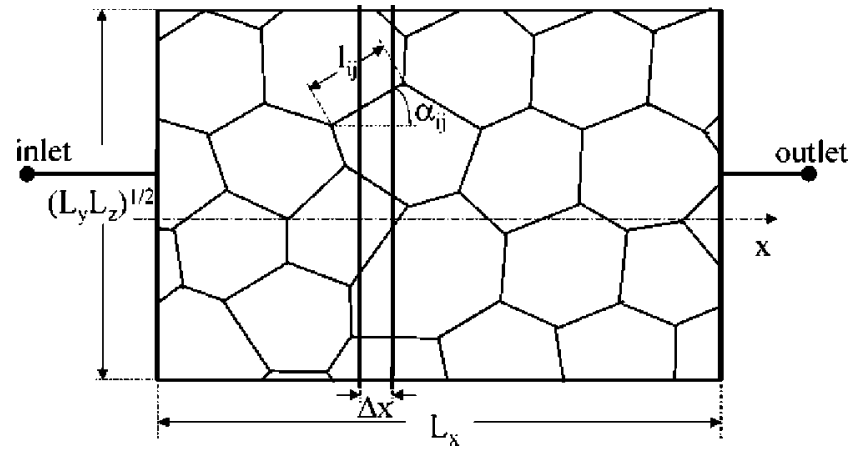

FIG. 1. Schematic representation of a network shorted with parallel sheets of infinite conductivity. The resistance of the truncated channel $(i, j)$ is equal to $\Delta x /\left[k_{i j}(l) \cos \alpha_{i j}\right]$.

\section{MAXIMAL AVERAGE CONDUCTIVITY OF A NETWORK}

On a macroscopic scale, the network can be seen as a continuous medium, a priori anisotropic, whose transport properties are described by an effective conductivity tensor $\underline{\underline{\sigma}}$ - the conductivity being defined as the permeability per unit length [two-dimensional (2D) network] or per unit area (3D network). The average conductivity associated with this tensor is defined as

$$
\sigma_{m}=\langle\mathbf{u} \cdot \underline{\underline{\sigma}} \cdot \mathbf{u}\rangle,
$$

where the brackets indicate that the term inside is averaged in all the directions of unit vector $\mathbf{u}$. This parameter can be simply related to the trace of $\underline{\underline{\sigma}}$ and the dimension of space $d$ [6] by

$$
\sigma_{m}=\frac{1}{d} \operatorname{Tr}[\underline{\underline{\sigma}}]
$$

The average conductivity characterizes the ability of the network to transport the physical quantity associated with it in all the directions. We shall show the existence of an upper bound for $\sigma_{m}$, which can be expressed as a function of the channel permeability and channel length distributions:

$$
\sigma_{m} \leqslant \frac{1}{d} \sum_{(i, j)} \frac{l_{i j} \bar{k}_{i j}}{L^{d}} .
$$

$L^{d}$ is the hypervolume of the network on which the conductivity tensor is defined, and $\bar{k}_{i j}$ is the average permeability of the channel $(i, j)$ defined by

$$
\bar{k}_{i j}=\frac{1}{l_{i j}} \int_{0}^{l_{i j}} k_{i j}(l) d l .
$$

We present here the demonstration for a three-dimensional network $(d=3)$. We first study the case of a network made of straight pipes: imagine that this network is shorted with thin parallel sheets of infinite conductivity, perpendicular to the direction $x$ of the applied potential difference and separated from each other by $\Delta x$, as in Fig. 1 ( $\Delta x$ has to be small compared to the typical channel length, but large compared to the typical channel diameter, so the flow lines in a given channel are mostly parallel to its axis). According to the monotonicity law, the conductivity of this shorted network is higher than the conductivity of the original one. Furthermore, the potential is uniform on each sheet and the resistance $\Delta R(x)$ of the network slice at position $x$ corresponds to the parallel association of the truncated channels that it contains. Since the resistance corresponding to a given truncated channel $(i, j)$ is equal to $\Delta x /\left[k_{i j}(l) \cos \alpha_{i j}\right]$, where $\alpha_{i j}$ is the angle between the channel $(i, j)$ and the axis $x$ (the angle is defined such that $\cos \alpha_{i j} \geqslant 0$ ), we have

$$
\frac{1}{\Delta R(x)}=\sum_{(i, j)} P\left(x ; x_{i}, x_{j}\right) \frac{k_{i j}(l) \cos \alpha_{i j}}{\Delta x},
$$

where the sum is carried out on all the channels of the network by introducing the function $P\left(x ; x_{i}, x_{j}\right)$ which take the value 1 if the channel $(i, j)$ is intersected by the equipotential plane passing by $x$ (i.e., if $x$ is between $x_{i}$ and $x_{j}$ ) and 0 otherwise. The total resistance is given by the sum of the slice resistances. Using the fact that the product of the average of a set of positive values $\left\{f_{1}, f_{2}, \ldots, f_{N}\right\}$ by the average of their inverses is always greater or equal to 1 ,

$$
\left(\frac{1}{N} \sum_{k=1}^{N} f_{k}\right)\left(\frac{1}{N} \sum_{k=1}^{N} \frac{1}{f_{k}}\right) \geqslant 1,
$$

and passing to the continuum limit, we obtain a lower bound for the resistance of the shorted network:

$$
\frac{1}{R_{x}} \leqslant \int_{0}^{\infty} \sum_{(i, j)} P\left(x, x_{i}, x_{j}\right) k_{i j}(l) \cos \alpha_{i j} \frac{d x}{L_{x}^{2}} .
$$

We can switch the sum and integral of this expression, and it follows after integration that

$$
\sigma_{x}^{(s)} \leqslant \frac{1}{L^{3}} \sum_{(i, j)} \bar{k}_{i j} l_{i j} \cos ^{2} \alpha_{i j},
$$

where the conductivity of the shorted network in the $x$ direction is defined by $\sigma_{x}^{(s)}=L_{x} / L_{y} L_{z} R_{x}, L_{y}$ and $L_{z}$ being the network lengths in directions $y$ and $z$, and $L^{3}=L_{x} L_{y} L_{z}$, the volume on which the conductivity is defined. Using the same arguments in the two other directions and the fact that the sum of the squared direct cosines is equal to 1 , we see that inequality (6) is true for a network made of straight channels. It is clear that the average conductivity of a network with curved channels is bounded, too: in this case, we can build a new network by keeping the junctions fixed in their positions but linked with straight channels. Let $l_{i j}^{\prime}$ be the length of the straight channel $(i, j)$. We can choose its local permeability $k_{i j}^{\prime}(l)$ to be equal to the local permeability of the corresponding curved channel on an arbitrary portion $l_{i j}^{\prime}$ of its lengthsay, $k_{i j}^{\prime}(l)=k_{i j}(l)$ for $0 \leqslant l \leqslant l_{i j}^{\prime}$. So the resistance of the straight channel, equal to $\int_{0}^{l_{i j}^{\prime}}\left[d l / k_{i j}^{\prime}(l)\right]$, is lower than the original curved channel. We know the conductivity $\sigma_{m}^{\prime}$ of such a network is bounded: 


$$
\sigma_{m}^{\prime} \leqslant \frac{1}{3} \sum_{(i, j)} \frac{l_{i j}^{\prime} \bar{k}^{\prime}{ }_{i j}}{L^{3}}
$$

On the one hand, the conductivity of this network is higher than the conductivity $\sigma_{m}$ of the original network (from the monotonicity law), and on the other hand, $l_{i j}^{\prime} \bar{k}_{i j}^{\prime} \leqslant l_{i j} \bar{k}_{i j}$, so inequality (6) holds for a network made of curved channels as well.

\section{OPTIMIZING TRANSPORT}

In addition to the existence and the expression of an upper bound for the average conductivity, we show that the average conductivity reaches the upper bound if and only if the following three conditions are satisfied.

(a) Each channel has a uniform local permeability along the channel $k_{i j}(l)=\bar{k}_{i j}=k_{i j}$.

(b) All the channels are straight.

(c) Every junction ( $i$ ) between channels satisfies $\sum_{j} k_{i j} \mathbf{e}_{i j}=0$, where $\mathbf{e}_{i j}$ are outward-pointing unit vectors in the directions of adjoining channels.

Note that the last condition is similar to a force balance equation at each vertex, the weight of the force pulling along a channel being proportional to its permeability. Furthermore, it is worth noting that the three conditions are independent of the connectivity of the junctions. As an illustrative example of this property, the periodic square, hexagonal, and triangular networks built with a same set of channels have the same conductivity.

Necessity of the conditions. Let us suppose that the conductivity of the network is equal to its maximal value

$$
\sigma_{m}=\frac{1}{d} \sum_{(i, j)} \frac{l_{i j} \bar{k}_{i j}}{L^{d}},
$$

and let us make some infinitesimal changes in the network structure. First, suppose we vary the local permeability of a given channel $(i, j)$ by a small amount $\delta k_{i j}(l)$, without altering its length $l_{i j}$ or its average permeability $\bar{k}_{i j}$, so

$$
\int_{0}^{l_{i j}} \delta k_{i j}(l) d l=0 \text {. }
$$

Since $l_{i j}$ and $\bar{k}_{i j}$ remain constant, the corresponding variation $\delta \sigma_{m}$ of the conductivity is zero. But obviously we can choose a variation $\delta k_{i j}(l)$ such that the resistance of the channel is increased:

$$
\int_{0}^{l_{i j}} \delta\left(\frac{1}{k_{i j}(l)}\right) d l \geqslant 0 .
$$

In order for the monotonicity law to be satisfied, the variation of the resistance has to be zero, which can be mathematically expressed as

$$
\int_{0}^{l_{i j}}\left(-\frac{1}{k_{i j}^{2}(l)}+\lambda\right) \delta k_{i j}(l) d l=0,
$$

where $\lambda$ is a Lagrange multiplier. Since this equality has to be true for any variation $\delta k_{i j}(l)$, it follows that $k_{i j}(l)$ has to be uniform along the channel, and so condition (a) is indeed required.

The necessity of conditions (b) and (c) is proved by means of arguments similar to those used in our previous work [3]: first, imagine that we change the length of a given channel $(i, j)$ of a network for which condition (a) is satisfied, the positions of all junctions and the lengths of all the other channels remaining unaltered. To this variation of length $\delta l_{i j}$ corresponds a variation of the conductivity:

$$
\delta \sigma_{m}=\frac{1}{L^{d} d} k_{i j} \delta l_{i j}
$$

implying that $\delta \sigma_{m}$ and $\delta l_{i j}$ have the same sign. However, if the length of the channel is increased $\left(\delta l_{i j} \geqslant 0\right)$, the resistance of the channel is increased too, and it follows from the monotonicity law that the conductivity can only decrease $\left(\delta \sigma_{m} \leqslant 0\right)$. So the variation of the length has be zero at first order, implying the necessity of condition (b).

Now, imagine a network for which conditions (a) and (b) are satisfied. In the previous section, we showed the existence of an upper bound of the conductivity in the direction $x$ for a network made of straight channels, by using two successive inequalities: first, the conductivity of such a network is lower than the conductivity of the same network intersected with zero resistance sheets; second, the shorted network conductivity itself is bounded, using the fact that the equivalent resistance of $N$ resistive elements in series arrangement is greater or equal to the equivalent resistance of the same resistive elements in parallel arrangement times $N^{2}$ [inequality (9)]. So in order to get the exact upper bound, these two inequalities have to become strict equalities. The first one implies that the presence of sheets does not modify the distributions of potentials, and so the potential in the channels is a function of $x$ only. To see this, increase progressively the resistance of the sheets up to infinity (which corresponds to the initial network). From the monotonicity law, this can only decrease the conductivity of the network. The only way for the conductivity to stay at its maximum value when increasing the resistance of each sheet is by having no current through them, and so the potential in the channels is a function of $x$ only. The second equality requires that the resistance of every slice (of equal thickness) is the same, or equivalently, the resistance of a slice of arbitrary thickness $x$ is simply proportional to $x$. Hence the potential is indeed linear in $x$. Examination of flow conservation at a vertex in such a potential immediately leads to the condition

$$
\sum_{j} k_{i j} \cos \alpha_{i j} \operatorname{sgn}\left(x_{i}-x_{j}\right)=0
$$

[the term $\operatorname{sgn}\left(x_{i}-x_{j}\right)$ is introduced in order to satisfy $\cos \alpha_{i j}$ $\geqslant 0]$. This equality is nothing but the projection of condition (c) on the axis $x$. Since the same argument holds in the two other directions, we prove that condition (c) is indeed required.

Sufficiency of the conditions. Now consider a network for which conditions (a), (b), and (c) are satisfied and suppose that a potential difference $U_{x}$ is applied between the two regarding faces orthogonal to $x$. Let us show that the trial 
potential function defined as $\phi=-\left(U_{x} / L_{x}\right) x$ is the physical solution. We first check that the flow is conserved at each junction under the stated conditions: the current in the straight channel $(i, j)$ is given by

$$
I_{i j}=-k_{i j} \nabla \phi \cdot \mathbf{e}_{i j}=k_{i j} \frac{U_{x}}{L_{x}} \mathbf{e}_{x} \cdot \mathbf{e}_{i j},
$$

so

$$
\sum_{j} I_{i j}=\frac{U_{x}}{L_{x}} \mathbf{e}_{x} \cdot \sum_{j} k_{i j} \mathbf{e}_{i j}=0 .
$$

The trial potential function also satisfies the boundary conditions and so is the correct physical solution. Now we can show that the average conductivity is equal to the upper bound. The potential is uniform on planes perpendicular to the direction $x$, and so the system is unaltered when intersected by thin parallel sheets of infinite conductivity orthogonal to that direction. We previously calculated the elementary resistance $\Delta R(x)$ of a slice of thickness $\Delta x$ at position $x$ for such a network [Eq. (8)]. The global flow rate $I_{x}$, the elementary resistance $\Delta R(x)$, and the elementary potential difference $\Delta \phi$ across the slice are related by

$$
\frac{\Delta \phi}{\Delta x}=\frac{\Delta R}{\Delta x} I_{x} .
$$

But here both the potential gradient and the global current are independent of $x$ and so is $\Delta R / \Delta x$. Using Eq. (8) and integrating in the $x$ direction, we finally get the expression of the conductivity in the $x$ direction:

$$
\sigma_{x}=\frac{1}{L^{d}} \sum_{(i, j)} k_{i j} l_{i j} \cos ^{2} \alpha_{i j} .
$$

This expression corresponds indeed to the upper bound of the conductivity along $x$, as expressed in Eq. (11). The same reasoning can be applied for the conductivity in the two other directions, and so the sufficiency of conditions (a), (b), and (c) is proved.

Let us apply the preceding results to two very common flow profiles-namely, plug flow and Poiseuille flow. Many transport phenomena through pipes are described by plug flow, such as the flow of fluids in porous conducts, the flow of heat by conduction, the electrical current, or diffusive flow. All these flows are engendered by a gradient of a potential function (such as pressure, temperature, electric potential, material concentration), and the permeability for all these situations is directly proportional to the cross-sectional area. Moreover, if the conductivity has the same value $\sigma_{0}$ for all pipes (so $k_{i j}=\sigma_{0} s_{i j}$ ), the upper bound of the average conductivity simplifies to

$$
\sigma_{m} \leqslant \frac{1}{d} \sigma_{0} \varepsilon,
$$

where $\varepsilon$ is the volume fraction of the continuous phase. As a consequence, the maximal conductivity does not depend on the permeability and length channel distributions, but only on the total amount of material that the network contains [there exists a universal upper bound of the quantity $\left(\sigma_{m} / \varepsilon\right)$,

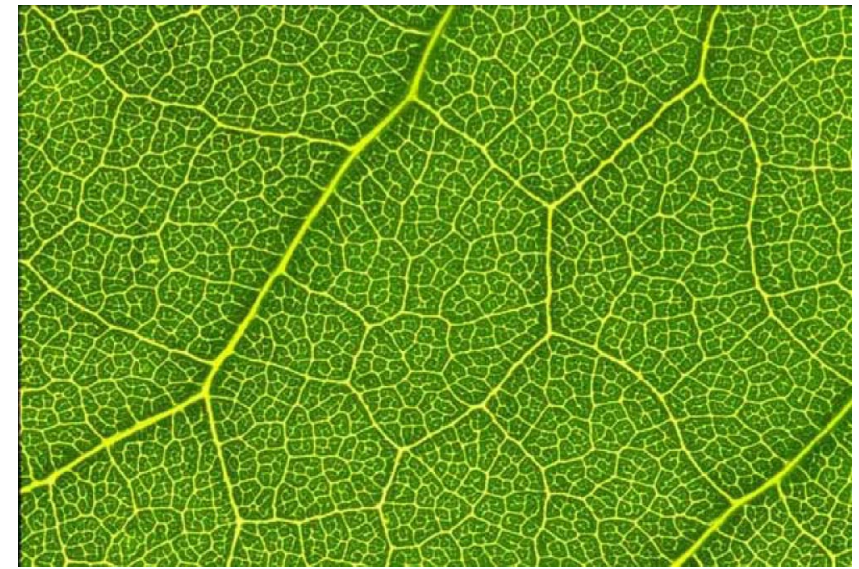

FIG. 2. Netted venation of a leaf $(4.82 \mathrm{~mm} \times 3.26 \mathrm{~mm})$. The angles between adjacent veins are correlated to their cross-section areas [10].

the average conductivity per unit volume of material]. Note that the expression of the upper bound is equivalent to the Hashin-Shtrikman bound for the electrical conductivity of a heterogenous material in the limit of small volume fraction of the conducting phase $[7,8]$.

Condition (c) leads to different optimal structures depending on the kind of flow profile in the channels. In the case of a plug flow profile with idential conductivity $\sigma_{0}$, this condition becomes

$$
\sum_{j} s_{i j} \mathbf{e}_{i j}=0 .
$$

In the case of Poiseuille flow, the permeability varies like the square of the cross-sectional area. Thus, if we assume again the same conductivity $\sigma_{0}$ for every pipe, condition (c) becomes

$$
\sum_{j} s_{i j}^{2} \mathbf{e}_{i j}=0 .
$$

As a concluding remark, it is worth noting that some natural networks, like leaf venation [10,11], have a very-welldefined structure, in the sense that the angles between adjacent veins are correlated to their cross-sectional areas (see Fig. 2). This fact presumably corresponds to some optimization principle. The conditions for transport optimization presented here may give an explanation for these typical patterns. In the particular case of leaf venation, veins are composed of a complex tangle of smaller tubes [9]; hence, we may expect relation (24) rather than (25) to be satisfied (although veins are not fully impermeable). Alternatively, this correlation between angles and cross-section areas could be explained by the optimization of the mechanical stability of the leaf $[12,13]$. Experimental study of the leaf venation structure is subject to a current investigation to be compared with the transport optimization criteria presented in this paper.

\section{ACKNOWLEDGMENT}

M. D. thanks H. A. Stone for encouraging this research. 
[1] M. Zamir, J. Theor. Biol. 62, 227 (1976).

[2] M. A. Changizi and C. Cherniak, Can. J. Physiol. Pharmacol. 78, 603 (2000).

[3] M. Durand, J.-F. Sadoc, and D. Weaire, Proc. R. Soc. London, Ser. A 460, 1269 (2004).

[4] J. W. S. Rayleigh, Collected Scientific Papers (Cambridge University Press, Cambridge, England, 1899), Vol. 1, pp. 3375.

[5] J. H. Jeans, The Mathematical Theory of Electricity and Magnetism, 5th ed. (Cambridge University Press, Cambridge, England, 1925).

[6] A priori $\sigma_{m}$ can be a function of the trace and the determinant of $\underline{\underline{\sigma}}$, which are the two invariants of a symmetrical secondorder tensor, but using the additivity property of tensors and dimensional analysis, it follows that $\sigma_{m}$ can only depend on the trace, in a linear way. The prefactor $1 / d$ comes naturally by studying the case of an isotropic network.

[7] Z. Hashin and S. Shtrikman, J. Appl. Phys. 35, 3125 (1962).

[8] S. Torquato, Random Heterogeneous Materials (SpringerVerlag, New York, 2002).

[9] A. H. de Boer and V. Volkov, Plant, Cell Environ. 26, 87 (2003).

[10] S. Bohn, B. Andreotti, S. Douady, J. Munzinger, and Y. Couder, Phys. Rev. E 65, 061914 (2002).

[11] Y. Couder, L. Pauchard, C. Allain, M. Adda-Beddia, and S. Douady, Eur. Phys. J. B 28, 135 (2002).

[12] A. Roth-Nebelsick, D. Uhl, V. Mosbrugger, and H. Kerp, Ann. Bot. (London) 87, 553 (2001).

[13] M. Durand (unpublished). 